\title{
Continuous-time quantum walks on spatially correlated noisy lattices
}

\author{
Matteo A. C. Rossi, ${ }^{1,}$ Claudia Benedetti, ${ }^{1}$ Massimo Borrelli, ${ }^{2}$ Sabrina Maniscalco, ${ }^{2,3}$ and Matteo G. A. Paris ${ }^{1}$ \\ ${ }^{1}$ Quantum Technology Lab, Dipartimento di Fisica, Università degli Studi di Milano, I-20133 Milano, Italy \\ ${ }^{2}$ Turku Centre for Quantum Physics, Department of Physics and Astronomy, University of Turku, FI-20014 Turun yliopisto, Finland \\ ${ }^{3}$ Center for Quantum Engineering, Department of Applied Physics, Aalto University School of Science, FI-00076 Aalto, Finland
}

(Received 6 July 2017; published 4 October 2017)

\begin{abstract}
We address memory effects and diffusive properties of a continuous-time quantum walk on a one-dimensional percolation lattice affected by spatially correlated random telegraph noise. In particular, by introducing spatially correlated time-dependent fluctuations in nearest-neighbor hopping amplitudes, we describe random domains characterized by global noise. The resulting open dynamics of the walker is then unraveled by an ensemble average over all the noise realizations. Our results show that time-dependent noise assisted by spatial correlations leads to strong memory effects in the walker dynamics and to robust diffusive behavior against the detrimental action of uncorrelated noise. We also show that spatially correlated classical noise enhances localization breaking, thus making a quantum particle spread on longer distances across the lattice.
\end{abstract}

DOI: 10.1103/PhysRevA.96.040301

Introduction. Continuous-time quantum walks (CTQWs) describe the free evolution of quantum particles on $N$-vertex graphs. They have been subject of intense studies, both theoretical and experimental, as they have proven useful for several applications, ranging from universal quantum computation [1], to search algorithms [2,3], quantum transport [4,5], quantum state transfer [6], and energy transport in biological systems [7].

Given their relevance in applications, a realistic description of the dynamics of quantum walkers should take into account those sources of noise and imperfections that might jeopardize the discrete lattice on which the CTQW occurs. While the effects of both disorder and dynamical fluctuations have been analyzed in the recent past [8-13], the consequences of spatially correlated noise on the dynamics of the walker are still, to the best of our knowledge, an unexplored territory.

In this Rapid Communication, we address the effects of spatially correlated noise by studying the most relevant dynamical features of a one-dimensional (1D) CTQW affected both by time- and space-dependent fluctuations. As for the former, the hopping amplitudes are assumed to fluctuate in time as a random-telegraph noise (RTN) inducing dynamical percolation, which results in a stochastic time-dependent Hamiltonian. This model has been studied in Ref. [10] in connection to particle localization and memory effects in the open dynamics of the walker. Here, we take it a step further and introduce random spatial correlations as follows: If two adjacent hopping links are subject to spatially correlated fluctuations, then they are affected by the same RTN time evolution. This will lead to the formation of percolation domains within which the tunneling amplitudes evolve according to the same stochastic noise. On a global scale, because of these spatial correlations, the hopping fluctuations will be synchronized domainwise. Overall, this is perhaps the simplest type of space dependency that one may introduce to the 1D CTQW, as it does not interfere with the local time-dependent part of the noise. The two sources of noise correlations may indeed be treated

\footnotetext{
*matteo.rossi@unimi.it
}

independently. At the same time, the model allows one to describe the formation of spatial domains and to address the percolation effect.

In turn, the motivation for introducing this extra ingredient is twofold. First, if one aims at a more realistic description of any experimental implementation of a CTQW, sources of noise should be accounted for. This is especially important when studying transport properties in disordered systems in which localization, let it be Anderson or many-body [14-16], represents an obvious obstacle. A renewed interest in this field has spurred deep investigations in highly engineered experimental setups, such as cold atoms in optical lattices $[17,18]$, in which complex noise might be efficiently implemented [19]. The second aspect concerns the question of whether the introduction of spatially correlated noise might result in improving certain dynamical features, such as slowing down decoherence or even the enhancement of quantum properties. In this respect, memory effects are of primary importance, as they have been shown to improve the performances of numerous protocols in quantum information [20-23] and quantum metrology [24,25]. They also play a key role in quantum thermodynamics [26] and measurement theory [27]. However, non-Markovian dynamics has been so far widely investigated and understood by focusing on the time domain [28], e.g., by inspecting quantities such as correlation functions at different times and the spectral densities of certain environments. It is this not obvious how, and whether, introducing spatially dependent noise might affect memory effects of a given dynamical map.

Our findings shed light on the effect of space-correlated dynamical noise on a quantum map, in terms of both memory effects and kinetic quantities, such as diffusion and velocity. As a matter of fact, spatially correlated noise results in stronger memory effects in the dynamics and it partly suppresses the localization induced by its randomness, allowing the walker to spread further and faster across the lattice.

The model. We consider a 1D lattice of $N$ sites and one particle (walker) freely moving across it. The Hamiltonian $H$ describing the walker's dynamics can be expanded in the single-particle localized orthonormal basis $\{|j\rangle\}$ with $j=1, \ldots, N$. If we introduce time-dependent stochastic 


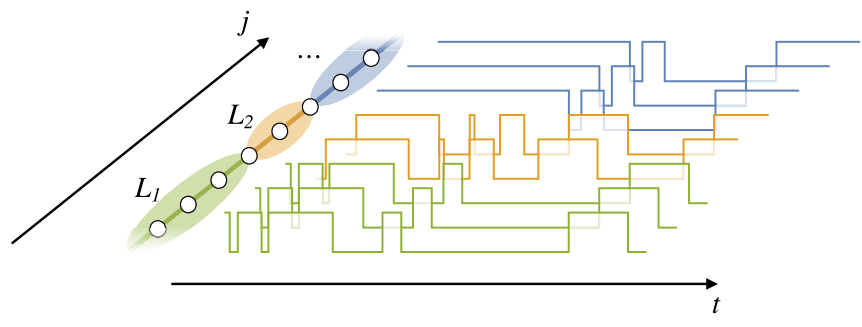

FIG. 1. Schematic representation of the random spatial domains $\left\{L_{1}, L_{2}, \ldots, L_{M}\right\}$ for a single realization of the noise, generated according to Eq. (3) and of average length $\bar{L}_{p}$. Tunneling amplitudes within the same domain fluctuate synchronously in time and according to the same stochastic process. Different domains evolve independently from each other.

fluctuations on the hopping amplitudes, the time-dependent Hamiltonian $H(t)$ reads

$$
H(t)=-\sum_{j}\left[v_{0}+v g_{j}(t)\right](|j\rangle\langle j+1|+| j+1\rangle\langle j|),
$$

in which $v_{0}$ is the uniform hopping amplitude between nearest-neighbor sites, $v$ is the noise strength, and $\left\{g_{j}(t)\right\}_{j}$ are independent RTN processes that jump between \pm 1 according to the switching rate $\gamma$.

We now introduce spatial correlations in the noisy Hamiltonian (1) as follows. We assume that two adjacent links of the lattice can be noise correlated with a certain probability $p$. Formally, this translates to the following autocorrelation function,

$$
\left\langle g_{j}(t) g_{k}(0)\right\rangle= \begin{cases}\propto e^{-2 \gamma t}, & \text { if } j, k \text { correlated } \\ 0, & \text { otherwise. }\end{cases}
$$

For a single noise realization, these spatial correlations will form $M$ domains of lengths $\left\{L_{1}, L_{2}, \ldots, L_{M}\right\}$, corresponding to $M$ independent noise evolutions $\left\{g_{1}(1), g_{2}(t), \ldots, g_{M}(t)\right\}$, respectively, as shown in Fig. 1. The distribution of the domains is random and different for each noise realization: The probability $P_{M}$ of having $M$ domains in a particular noise realization is described by a binomial distribution

$$
P_{M}=\left(\begin{array}{l}
N-1 \\
M-1
\end{array}\right)(1-p)^{M-1} p^{N-M},
$$

which corresponds to the following average domain length $\bar{L}$ (as a function of $p$ ), $\bar{L}_{p}=\frac{p^{N}-1}{p-1}$. By continuity, we define $\bar{L}_{1}=\lim _{p \rightarrow 1} \bar{L}_{p}=N$. In this case, there is a single noise domain that spans the whole lattice.

So far, the amplitude of the fluctuations $v$ has been considered a free parameter of the strength of the noise. Here, we are interested in the effect of noise space and time correlations per se, rather than in the noise strength. Thus, we set this parameter to $v=v_{0}$, meaning that, from now on, we are only going to consider percolation noise: The local hopping amplitudes can switch between 0 and $2 v_{0}$ [29], resulting in links that are created and destroyed randomly in time, according to the statistics of the RTN process. Quite obviously, this analysis can be carried out for any value of $v$.

For each noise realization, the system time evolution is ruled by the operator $U(t)=\mathcal{T} e^{-i \int_{0}^{\tau} d \tau H(\tau)}$. The open dynamics of the walker is unraveled by computing the ensemble average of the unitary dynamics over all possible realizations,

$$
\bar{\rho}(t)=\Lambda_{t} \rho_{0}=\left\langle U(t) \rho_{0} U^{\dagger}(t)\right\rangle_{\{g(t)\}},
$$

where $\langle\cdot\rangle_{\{g(t)\}}$ indicates the average taken over an (in principle) infinite number of implementations of the sets $\left\{g_{1}(1), g_{2}(t), \ldots, g_{M}(t)\right\}$ and $\rho_{0}$ is the (fixed) initial state of the walker. Needless to say, whenever the solution to Eq. (4) is analytically out of reach, one can only numerically approximate this ensemble average with a finite number of noise realizations $R$. In this case we talk about undersampling [30] and the true dynamics (4) can be recovered only in the limit $R \rightarrow \infty$. For all the quantities computed in this work, the size of the noise sample is $R=10000$, which guarantees statistical robustness of our results. The code for simulating the dynamics is reported and explained in the Supplemental Material [31].

Non-Markovianity of the dynamical map. As previously mentioned, the noise-averaged dynamics of the walker can no longer be described by the Schrödinger equation and one has to resort to the machinery of open quantum systems. In this respect, a relevant question is whether the open dynamics of the walker is memoryless, i.e., Markovian, or non-Markovian. In Ref. [10], memory effects in the dynamics of the walker in the presence of spatially uncorrelated RTN were investigated for some selected initial states, leading to the conclusion that decreasing the switching rate $\gamma$ enhances the memory effects. That scenario corresponds to noise domains of average length $\bar{L}=1$ and therefore it is a special case study of the more general model introduced in this Rapid Communication. Intuitively, since the non-Markovian dynamics is intrinsically connected to the time dependency of the environment correlation functions, we can expect that whenever the spatially uncorrelated noise is Markovian, it will also be Markovian in the spatially correlated noise case. This is simply because, as mentioned previously, the spatial correlations in the noise do not interfere with the RTN itself but they only assist it. However, if memory effects are present already in the spatially uncorrelated scenario, it is not obvious a priori how long-range correlated noise with $\bar{L}>1$ will affect the non-Markovianity of the quantum map. Similarly to Ref. [10], we use the trace-distance-based Breuer-Laine-Piilo (BLP) [32] approach to characterize memory effects in the open dynamics of the walker. The trace distance between two quantum states $\rho_{1}$ and $\rho_{2}$ is defined as $D(t)=D\left(\rho_{1}(t), \rho_{2}(t)\right)=\frac{1}{2} \| \rho_{1}(t)-$ $\rho_{2}(t) \|$, where $\|A\|=\operatorname{Tr}\left[\sqrt{A^{\dagger} A}\right], \rho_{1(2)}(t)=\Lambda_{t} \rho_{1(2)}$, and $\Lambda_{t}$ denotes a dynamical quantum map. For a Markovian map, $D(t)$ monotonically decreases in time for any initial pair of states. Therefore, a violation of such a constraint signals the presence of memory effects or, equivalently, a non-Markovian dynamics. A quantifier of memory effects can be defined by integrating the time derivative of $D(t)$ over the time intervals where the trace distance has revivals, i.e., $\dot{D}(t)=$ $d D(t) / d t>0$, and then maximizing over all the possible pairs of initial states. Computationally, this translates to evaluating the following quantity,

$$
\mathcal{N}=\max _{\rho_{1}, \rho_{2}} \int_{\dot{D}(t)>0} d t \frac{d}{d t} D\left(\Lambda_{t} \rho_{1}, \Lambda_{t} \rho_{2}\right),
$$


in which $\Lambda_{t}$ is the dynamical map (4). The above quantity is, in practice, nearly impossible to compute exactly because it involves a state optimization procedure and only few analytically treatable cases are known in the literature [20]. Nonetheless, it does provide a rather intuitive interpretation of memory effects in open systems and it still allows one to get insight into the behavior of memory effects by selecting some significant pairs of initial states.

Diffusion versus localization. The inverse participation ratio. In Ref. [10], the dynamics governed by Eq. (1) in the absence of spatially correlated noise was analyzed in detail, showing a transition from a diffusive to a localized regime as a function of the switching rate $\gamma$. Furthermore, depending on the strength of the noise, a quantum-to-classical transition was also observed for the fast noise case $(\gamma>1)$, resulting in a Gaussian probability distribution of the walker's state. Here, we aim at understanding the role of noise spatial correlations in the dynamical behavior of the walker. Specifically, we want to understand whether spatially correlated noise domains help the particle spread over the lattice or whether they instead favor localization. We quantify the extent of noise-induced localization by means of the inverse participation ratio (IPR) [33], defined as

$$
\mathcal{I}(t)=\sum_{j=1}^{N}\langle j|\bar{\rho}(t)| j\rangle^{2} .
$$

IPR is bounded between $1 / N$ and 1 with $\mathcal{I}(t)=\frac{1}{N}$, meaning complete delocalization, and $\mathcal{I}(t)=1$ corresponding to localization on a single site. The larger the IPR, the more localized is the particle. Using IPR we now investigate how the spatially correlated time-dependent noise affects the diffusive properties of the walker.

Results. We now present our results on the dynamical properties of the walker in a noisy, spatially correlated lattice. The evolution of the walker is obtained by randomly generating the domains and the noise realizations, computing the single realization unitary dynamics, and finally performing the ensemble average (4) for an $N=100$ lattice and for $R=10000$ iterations. First, we will focus on the non-Markovian character of the quantum map, then analyze the diffusive properties of the CTQW. As anticipated above, the maximization in Eq. (5) is a nearly impossible task for most physical systems. Because of our computational resources and the complexity of the model at hand, this case study is certainly no exception. However, we can still compute the integral in Eq. (5) for some relevant initial pairs of states and gain useful information regarding at least their dynamics. Since we are interested in the interplay between noise-induced localization and memory effects due to spatially correlated noise, we restrict our attention to pairs of initial states that are localized on adjacent sites and we compute the following quantity,

$$
n_{\tau}(\gamma, \bar{L})=\int_{\dot{D}(t)>0} d t \frac{d}{d t} D\left(\Lambda_{t} \rho_{N / 2}, \Lambda_{t} \rho_{1+N / 2}\right),
$$

for a fixed final time $\tau$, as a function of $\gamma$. In the above equation, $\rho_{j}=|j\rangle\langle j|$ and $\Lambda_{t}=\Lambda_{t}(\gamma, \bar{L})$ is the dynamical map computed via Eq. (4) that depends upon the value of the noise switching rate $\gamma$ and the average domains length $\bar{L}$. The

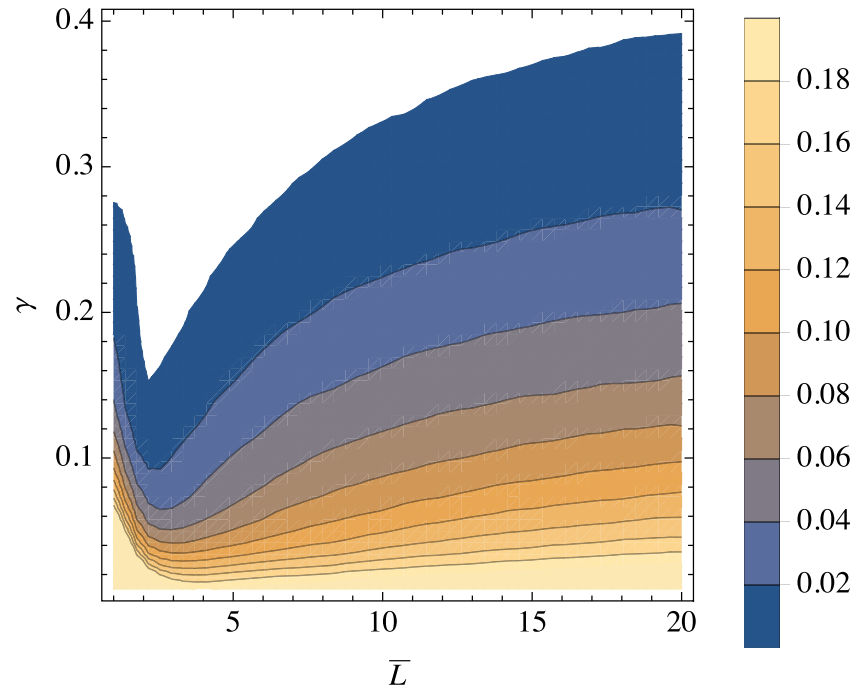

FIG. 2. Non-Markovianity $n_{\tau}(\gamma, \bar{L})$ as a function of the average domain length $\bar{L}$ and switching rate $\gamma$ for percolation noise. The selected initial states are $|N / 2\rangle$ and $|N / 2+1\rangle$ with $N=100$ and $v_{0} \tau=20$. In the white region, $n_{\tau}(\gamma, \bar{L})=0$.

integral over time in Eq. (7) is up to the fixed time $\tau$. In Fig. 2, we display $n_{\tau}(\gamma, \bar{L})$ for an $N=100$ lattice and $\nu_{0} \tau=20$. We choose this truncation time to ensure that the tails of the walker wave function have not yet reached the boundaries of the lattice and therefore we need not worry about finite-sizeinduced memory effects. Here, we analyze a range of values for $\gamma$ that are known to generate non-Markovian dynamics, for the same initial states, in the case of noncorrelated RTN [10]. The striking feature we immediately notice is that, after a minimum located at $\bar{L} \approx 2$ and independent of $\gamma$, as the average domain length $\bar{L}$ is increased, the non-Markovian character evaluated through Eq. (7) also increases. Thus spatial correlations in the noise make memory effects stronger, at least for this set of initial states. An intuitive theoretical explanation of this behavior might be the following. The presence of domains with a typical length $\bar{L}$ is effectively equivalent to amplifying the single-link contribution to memory effects proportionally to the size of the domain. The walker experiences a smaller effective lattice of size $M$ with, however, a stronger average local disorder. We performed this calculation using increasingly separated localized initial states and found the exact same behavior, with the only difference being a smaller value of $n_{\tau}(\gamma, \bar{L})$.

In Fig. 3, we display the long-time IPR $\mathcal{I}\left(v_{0} \tau\right)$ as a function of $\gamma$ and $\bar{L}$ computed for the initially localized state $|N / 2\rangle$. This quantity is defined as the quasistationary value that the IPR reaches before boundary effects come into play. Interestingly, for a fixed $\gamma$, this has a maximum at $\bar{L}=1$, similarly to $n_{\tau}(\gamma, \bar{L})$, and decays fast as $\bar{L}$ increases. While uncorrelated slow noise tends to keep the walker localized around its initial position, spatial correlations break the localization and lead to a stronger diffusion of the wave function across the lattice. By increasing the value of the switching rate $\gamma$, the IPR becomes smaller as we approach memoryless and more diffusive dynamics. Therefore, while the presence of slow noise $(\gamma<1)$ tends to favor localization, by adding random spatial correlations to the very same noise we 


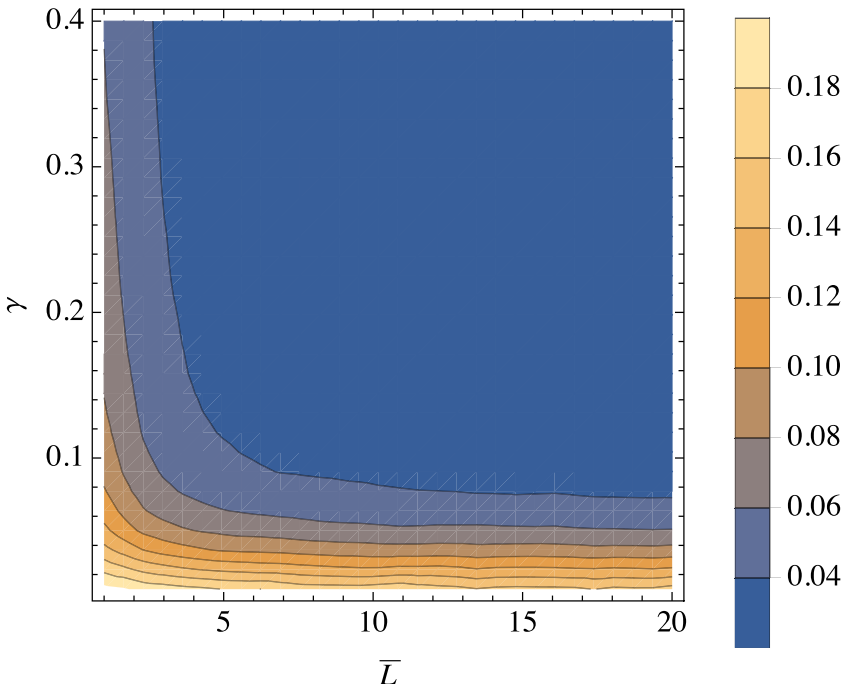

FIG. 3. Long-time value of the IPR as a function of average domain length $\bar{L}$ and switching rate $\gamma$ for percolation noise for the initial states $|N / 2\rangle$ with $N=100$ for $\nu_{0} \tau=20$.

can limit this effect and allow the walker to propagate through the lattice while still retaining memory effects in its dynamics. Overall, and perhaps quite unexpectedly, for a small fixed $\gamma$, a spatially correlated RTN tends to suppress localization while still enhancing memory effects.

To investigate transport properties in this setting we turn our attention to an initial Gaussian wave packet, equipped with an average momentum $k_{0}$ and spatial spread $\Delta$,

$$
|\mathcal{G}\rangle=\sum_{j=1}^{N}\left[\frac{1}{\sqrt{2 \pi \Delta^{2}}} e^{-\frac{\left(j-\frac{N}{2}\right)^{2}}{2 \Delta^{2}}}\right] e^{-i k_{0} j}|j\rangle .
$$

We study the behavior of both the IPR and the average momentum operator $\hat{p}=-i \nabla$, computed using the Born rule
$\langle\hat{p}(t)\rangle=\operatorname{Tr}[\bar{\rho}(t) \hat{p}]$, which represents the average quantum velocity at which the wave packet travels across the lattice. Figure 4 shows the time evolution of these two quantities for three different values of the switching rate $\gamma$ and different average domain lengths $\bar{L}$. In this case, the effects of the spatially correlated RTN become even clearer. The wavepacket momentum $\langle p\rangle$ (upper panel) decreases in time, until it eventually vanishes asymptotically, and this decay is faster for smaller values of $\gamma$, in agreement with Fig. 3. However, while space-uncorrelated noise leads to a faster reduction of $\langle\hat{p}\rangle$, spatial correlations in the RTN allow the wave packet to preserve momentum and travel longer across the lattice before stopping. In the limiting case of $\bar{L}=N$ (i.e., $p=1$ ), the average momentum $\langle\hat{p}\rangle$ is preserved, as in the noiseless case.

Similarly to the case studied above, the IPR (lower panel) generally decreases in time. However, there seems to exist a more complicated interplay between $\gamma$ and $\bar{L}$. For small $\gamma$ the IPR decays faster for larger values of $\bar{L}$, indicating that spatial correlations break the noise-induced localization, in agreement with our previous results. For larger switching rates $\gamma$, instead, the situation is quite the opposite: Strong spatial correlations prevent the particle distribution from spreading further, thus preserving the initial IPR, with the limiting case of $p=1$, i.e., $\bar{L}=N$ that gives the slowest possible decay.

Since the average momentum $\langle p\rangle$ decreases very slowly in time in this regime, the original wave packet can travel across the lattice, maintaining its original shape. This feature is the key ingredient for quantum transport and state transfer, where one wants a quantum state to evolve across a complex network, without losing its quantum properties, so that its quantum information content can be recovered from another point in the network.

Therefore, we have again evidence of how the introduction of space correlations in the noise helps preserve dynamical
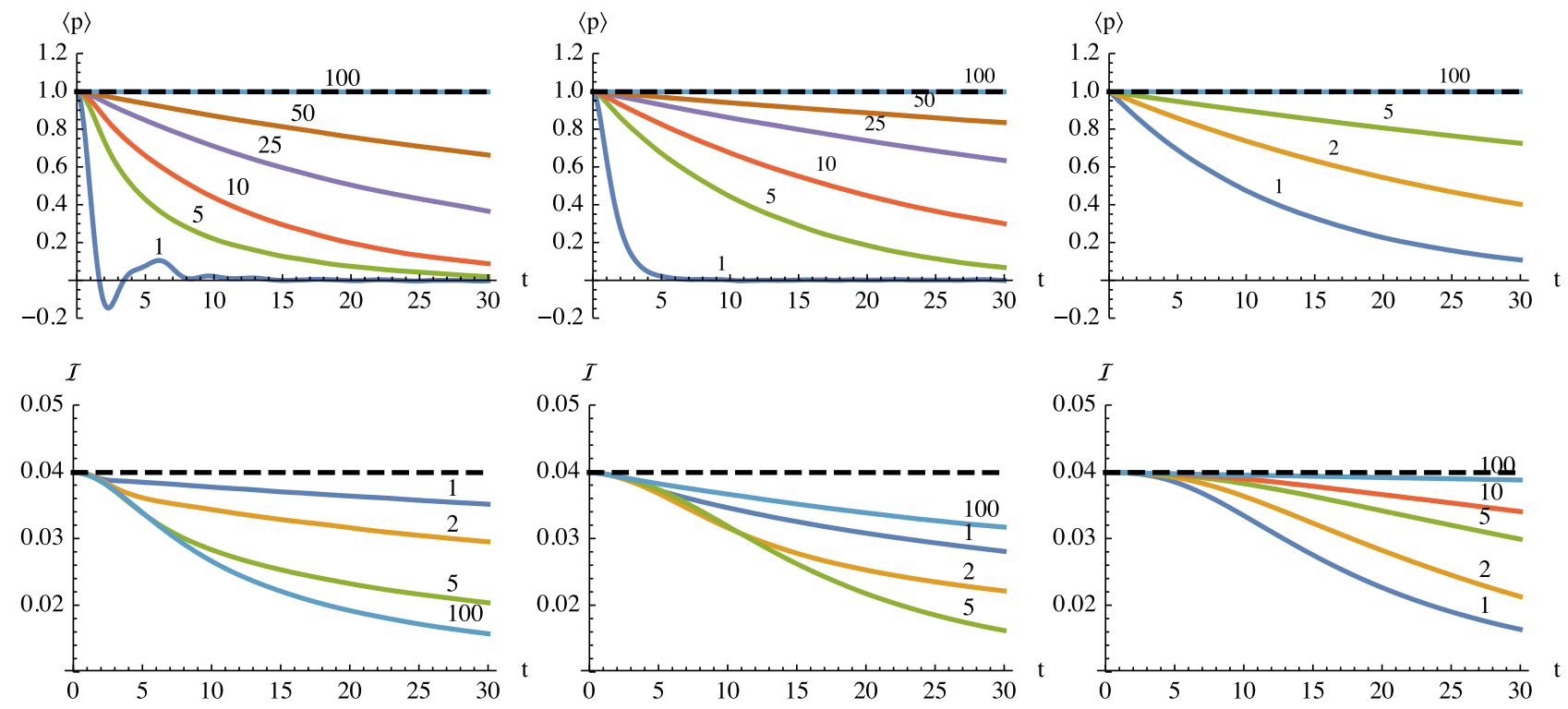

FIG. 4. Expectation value of the momentum operator $\langle p\rangle$ (top panels) and IPR $\mathcal{I}$ (bottom panels) as a function of time, for different average domain lengths $\bar{L}$, for $\gamma=0.1$ (left), 1 (center), and 10 (right), with lattice size $N=100$. The black dashed line indicates the noiseless case. The initial state is (8), with $k_{0}=\pi / 2, \Delta=10$. 
properties better than in the spatially uncorrelated case. This can surely be exploited to design protocols for state transfer and communication across networks.

Conclusions. We have addressed in detail the effects of spatial correlations on the dynamics of continuous-time quantum walks on noisy percolation lattices. Our model, which allows us to address memory effects and transport properties, is based on a stochastic time-dependent Hamiltonian, where the hopping amplitudes between adjacent nodes are described as local random-telegraph processes, which themselves show spatial correlations.

Our results show that classical spatial correlations in the noise make quantum features of the CTQW more robust. More specifically, we have provided evidence that the presence of strongly spatially correlated noise induces robust memory effects on the quantum map, as compared to the case of uncorrelated RTN. Furthermore, spatial correlations lead to localization breaking, i.e., make the walker able to spread over the network and to reach distant nodes while still undergoing non-Markovian dynamics. Finally, we have shown that spatially correlated RTN improves transport properties of an initially traveling Gaussian packet compared to the analog uncorrelated case.

Our analysis provides insight into the effects of spatially correlated noise on simple graphs and represents a step toward the understanding of the role of correlated fluctuations on complex networks, which, in turn, are extremely relevant to several quantum information and computation tasks, such as quantum algorithms, quantum communication, and models for realistic transport across distant nodes.

This work has been supported by EU through the collaborative H2020 project QuProCS (Grant Agreement No. 641277).
[1] A. M. Childs, Phys. Rev. Lett. 102, 180501 (2009).

[2] A. M. Childs and J. Goldstone, Phys. Rev. A 70, 022314 (2004).

[3] S. Chakraborty, L. Novo, A. Ambainis, and Y. Omar, Phys. Rev. Lett. 116, 100501 (2016).

[4] O. Mülken, V. Pernice, and A. Blumen, Phys. Rev. E 76, 051125 (2007).

[5] H. Bougroura, H. Aissaoui, N. Chancellor, and V. Kendon, Phys. Rev. A 94, 062331 (2016).

[6] D. Tamascelli, S. Olivares, S. Rossotti, R. Osellame, and M. G. A. Paris, Sci. Rep. 6, 26054 (2016).

[7] M. Mohseni, P. Rebentrost, S. Lloyd, and A. Aspuru-Guzik, J. Chem. Phys. 129, 174106 (2008).

[8] Y. Yin, D. E. Katsanos, and S. N. Evangelou, Phys. Rev. A 77, 022302 (2008).

[9] A. Schreiber, K. N. Cassemiro, V. Potoček, A. Gábris, I. Jex, and C. Silberhorn, Phys. Rev. Lett. 106, 180403 (2011).

[10] C. Benedetti, F. Buscemi, P. Bordone, and M. G. A. Paris, Phys. Rev. A 93, 042313 (2016).

[11] I. Siloi, C. Benedetti, E. Piccinini, J. Piilo, S. Maniscalco, M. G. A. Paris, and P. Bordone, Phys. Rev. A 95, 022106 (2017).

[12] Z. J. Li and J. B. Wang, Sci. Rep. 5, 13585 (2015).

[13] T. Chattaraj and R. V. Krems, Phys. Rev. A 94, 023601 (2016).

[14] P. W. Anderson, Phys. Rev. Lett. 18, 1049 (1967).

[15] D. Basko, I. Aleiner, and B. Altshuler, Ann. Phys. 321, 1126 (2006).

[16] R. Nandkishore and D. A. Huse, Annu. Rev. Condens. Matter Phys. 6, 15 (2015).

[17] I. Bloch, J. Dalibard, and W. Zwerger, Rev. Mod. Phys. 80, 885 (2008).
[18] M. A. Cazalilla, R. Citro, T. Giamarchi, E. Orignac, and M. Rigol, Rev. Mod. Phys. 83, 1405 (2011).

[19] F. Meinert, M. J. Mark, K. Lauber, A. J. Daley, and H.-C. Nägerl, Phys. Rev. Lett. 116, 205301 (2016).

[20] A. Karlsson, H. Lyyra, E.-M. Laine, S. Maniscalco, and J. Piilo, Phys. Rev. A 93, 032135 (2016).

[21] B.-H. Liu, X.-M. Hu, Y.-F. Huang, C.-F. Li, G.-C. Guo, A. Karlsson, E.-M. Laine, S. Maniscalco, C. Macchiavello, and J. Piilo, Europhys. Lett. 114, 10005 (2016).

[22] E.-M. Laine, H.-P. Breuer, and J. Piilo, Sci. Rep. 4, 04620 (2014).

[23] B. Bylicka, D. Chruściński, and S. Maniscalco, Sci. Rep. 4, 5720 (2014).

[24] Y. Matsuzaki, S. C. Benjamin, and J. Fitzsimons, Phys. Rev. A 84, 012103 (2011).

[25] A. W. Chin, S. F. Huelga, and M. B. Plenio, Phys. Rev. Lett. 109, 233601 (2012).

[26] B. Bylicka, M. Tukiainen, D. Chruściński, J. Piilo, and S. Maniscalco, Sci. Rep. 6, 27989 (2016).

[27] G. Karpat, J. Piilo, and S. Maniscalco, Europhys. Lett. 111, 50006 (2015).

[28] H.-P. Breuer, E.-M. Laine, J. Piilo, and B. Vacchini, Rev. Mod. Phys. 88, 021002 (2016).

[29] Z. Darázs and T. Kiss, J. Phys. A 46, 375305 (2013).

[30] M. A. C. Rossi, C. Benedetti, S. Cialdi, D. Tamascelli, S. Olivares, B. Vacchini, and M. G. A. Paris, arXiv:1705.05852.

[31] See Supplemental Material at http://link.aps.org/supplemental/ 10.1103/PhysRevA.96.040301 for the code for numerical simulation.

[32] H.-P. Breuer, E.-M. Laine, and J. Piilo, Phys. Rev. Lett. 103, 210401 (2009).

[33] M. Modugno, New J. Phys. 11, 033023 (2009). 